\title{
FRAGILIDADE E SUPORTE SOCIAL DE IDOSOS EM REGIÃO VULNERÁVEL: UMA ABORDAGEM EM UMA UNIDADE DE SAÚDE DA FAMÍLIA
}

\section{FRAILTY AND SOCIAL SUPPORT OLDER ADULTS' IN A VULNERABLE REGION: AN AP- PROACH IN A FAMILY HEALTH UNIT}

Karla Moura ${ }^{\mathrm{a}^{*}}$

Orcid: https://orcid.org/0000-0002-1946-9461

Isabela Thaís Machado de Jesus ${ }^{\mathrm{b}^{*}}$

Orcid: https://orcid.org/0000-0002-3752-8867
Ariene Angelini dos Santos Orlandi ${ }^{*}$

Orcid: https://orcid.org/0000-0002-3112-495X

Marisa Silvana Zazzetta ${ }^{\mathrm{d}^{*}}$

Orcid: https://orcid.org/0000-0001-6544-767X

karla.moura@live.com ${ }^{\mathrm{a}}$, isabela.machado1@gmail.com ${ }^{\mathrm{b}}$, arieneangelini@yahoo.com.br ${ }^{\mathrm{c}}$, marisam@ufscar.br ${ }^{\mathrm{d}}$

Universidade Federal de São Carlos

Data de submissão: 04/10/2019

Data de aceite $14 / 01 / 2020$

\section{RESUMO}

Introdução: Fragilidade é uma síndrome geriátrica prevalente nas pessoas idosas sendo acometida por diferentes fatores de risco. A ausência de suporte social parece ser fator de risco para a síndrome. Objetivo: Analisar a relação entre fragilidade e suporte social em idosos cadastrados em uma Unidade de Saúde da Família em uma região de vulnerabilidade social. Materiais e Métodos: Estudo transversal e quantitativo. Participaram do estudo pessoas com idade a partir de 60 anos ou mais, cadastrados em uma Unidade de Saúde da Família. Os participantes foram avaliados pela Escala de Fragilidade de Edmonton; Fragilidade Autorreferida; Tilburg Frailty Indicator e Medical Outcomes Study. Todos os aspectos éticos foram respeitados (Número de Parecer: 2.375.412). Resultados: Participaram do estudo 55 idosos sendo uma maioria homens $(57,10 \%)$, casados $(44,60 \%)$ e não aposentados $(55,40 \%)$. A fragilidade medida pelos instrumentos subjetivos mostrou-se prevalente na amostra estudada. O suporte social dos participantes apresentou uma média de 70,47 pontos. Quanto à análise de correlação, não houve relação entre as medidas de fragilidade e o suporte social. Conclusões: $\mathrm{O}$ estudo não apresentou relação entre fragilidade e suporte social. No entanto, rastrear a fragilidade e o suporte social em pessoas idosas pode direcionar estratégias preventivas pelas equipes de atenção primária à saúde com foco no cuidado de longa duração.

Palavras-chave: Idoso fragilizado; suporte social; atenção primária à saúde

\begin{abstract}
Background: Frailty is a geriatric syndrome prevalent in older people being affected by different risk factors. The lack of social support seems to be a risk factor for the syndrome. Objective: To analyze the relationship between frailty and social support in older adults enrolled in a Family Health Unit in a region of social vulnerability. Methods: Cross-section and quantitative study. The participants were evaluated by the Edmonton Frail Scale, Self-reported Frailty, Tilburg Frailty Indicator and Medical Outcomes Study. All ethical principles were respected (Opinion Number: 2.375.412). Results: Fifty-five older people participated in the study, most of them male (57.10\%), married (44.60\%) and non-retirees (55.40\%). The frailty measured by subjective instruments was prevalent in the sample studied. The participants' social support presented an average of 70.47 points. In relation to correlation analysis, there was no relationship between frailty measures and social support. Conclusions: The study was not showed a relation between frailty and social support. However, to screen frailty and social support in older people can direct preventive strategies by primary health care teams focusing on long-term care.
\end{abstract}

Keywords: Frailty older adult; social support; primary health care 


\section{Introdução}

A longevidade é, sem dúvida, uma conquista decorrente dos avanços tecnológicos e recursos de saúde, porém encontra-se em diferentes estágios no mundo mesmo em países com desenvolvimento econômico semelhante. Enquanto que em países desenvolvidos, o envelhecimento ocorreu associado às melhorias nas condições gerais de vida e em países em desenvolvimento, esse processo acontece sem que haja uma reorganização adequada tanto na área social como na área da saúde ${ }^{1}$.

Uma síndrome geriátrica que vem sendo observada com a longevidade é a fragilidade, caracterizada pela alta vulnerabilidade física em que expõe o indivíduo em diferentes estressores. Tem como principais fatores de risco a idade avançada, gênero feminino, baixa escolaridade, baixa renda, polifarmácia, ausência de suporte familiar e social, hábitos de vida pouco saudáveis, comprometimento cognitivo e hospitalização. Essa associação multifatorial compromete os mecanismos homeostáticos e a capacidade de reserva funcional de forma progressiva e lenta, podendo provocar desfechos negativos à saúde e por consequência ao óbito² .

A prevalência de fragilidade em idosos brasileiros, em recente revisão sistemática apontou variação entre 6,7 e $74,1 \%{ }^{3}$. Um estudo realizado com idosos cadastrados na proteção social básica em uma cidade do estado de São Paulo avaliou 247 idosos residentes em diferentes regiões com vulnerabilidade social segundo a Escala de Fragilidade de Edmonton e obteve uma prevalência de $36,8 \%$ de idosos frágeis. Houve correlação da fragilidade com a presença do suporte social ${ }^{4}$. Outro estudo com 377 idosos usuários da Estratégia de Saúde da Família no estado do Mato Grosso estimou a prevalência de fragilidade em $65,25 \%{ }^{5}$. Em um estudo, Llano et al (2017) avaliaram a fragilidade Autorreferida em 820 idosos residentes em áreas rurais no Rio Grande do Sul e identificaram que $43,4 \%$ dos idosos apresentaram fragilidade ${ }^{6}$.

Cesari et al $(2016)^{7}$ indicaram que condições socioeconômicas pobres e acesso a serviços de saúde deficientes durante a fase jovem e adulta influenciarão a condição de saúde na velhice.
A vulnerabilidade social, que expressa uma condição de pessoas ou famílias que estão em processo de exclusão social e é caracterizada por aspectos econômicos, culturais e sociais precários, quando interage com os aspectos biológicos do indivíduo, exerce um forte influxo sob o indivíduo, determinando seu modo de vida $^{8}$. Evidência na literatura científica aponta que idosos em condição de alta vulnerabilidade social apresentam condição de saúde mais fragilizada9 .

Avaliar a rede de apoio e a situação de vulnerabilidade social do indivíduo idoso é de suma importância. Alvarenga $(2011)^{10}$ salienta que investigar a composição familiar e a função de seus membros pode colaborar de forma significativa para o planejamento do cuidado ao idoso. O suporte social é fator capaz de proteger e promover a saúde e influencia diretamente na capacidade adaptativa e resiliência a estressores ${ }^{10}$.

Marques et al. (2011) ${ }^{11}$ classificaram o suporte social como formal ou informal, em que os sistemas formais são os serviços de atendimento ao idoso e o sistema informal ou rede de suporte social compreende as redes de relacionamentos familiar, amigos, relações de trabalho, inserção comunitária e participação social. Amaral et al $(2013)^{9}$ afirmam que "a fragilidade possa ser agravada devido à ruptura de laços sociais", ainda segundo os Autores manter o nível de integração social está inversamente associado à mortalidade.

Nesse contexto conhecer a dinâmica da relação da fragilidade com o suporte social e outros indicadores de saúde podem ser utilizados para determinar a efetividade e a eficiência de intervenções propostas ${ }^{2,12}$. Uma estratégia de enfrentamento para essa demanda seria instrumentalizar a atenção básica em relação à fragilidade uma vez que, segundo a Política Nacional de Saúde da Pessoa Idosa, ${ }^{13}$ a atenção à saúde da pessoa idosa tem como porta de entrada a Atenção Básica e deve ser responsável por todas as pessoas idosas que estão na sua área de abrangência, inclusive, aquelas que se encontram institucionalizadas. O objetivo deste trabalho foi analisar a relação entre fragilidade e suporte social em idosos cadastrados em uma Unidade de Saúde da Família em uma região de alta vulnerabilidade social. 


\section{Metodologia}

Tratou-se de um estudo transversal e exploratório com a utilização do método quantitativo de investigação. O estudo foi realizado no município de Piracicaba, que segundo o Instituto Brasileiro de Geografia e Estatística tem uma população estimada em 394.419 habitantes e $12 \%$ da população fazem referência às pessoas com 60 anos ou mais.

Foi realizada junto à Prefeitura do município de Piracicaba a identificação das regiões de maior vulnerabilidade e mapeamento de uma macroárea de saúde, em que a pesquisa foi realizada. Para a caracterização das áreas vulneráveis utilizouse o Índice Paulista de Vulnerabilidade Social $(2010)^{14}$ e pôde ser verificado que no município $21 \%$ da população encontrava-se em situação de média a muito alta vulnerabilidade social. Segundo a Prefeitura de Piracicaba - Secretária de Comunicação, as regiões de maior vulnerabilidade pertenciam aos grupos cinco e seis, sendo as regiões Oeste, Norte e Leste. A população estimada nessas regiões é de aproximadamente 54 mil pessoas cadastradas e atendidas por Unidade de Saúde da Família (USF).

De acordo com os dados obtidos, o estudo foi direcionado à população pertencente ao grupo seis, no bairro Tatuapé, USF Tatuapé 1, que contava com uma população de aproximadamente 1.300 famílias cadastradas e com membros idosos. Mediante isso, foram identificadas 197 pessoas idosas. Posteriormente, realizou-se um levantamento dos idosos residentes na região junto à equipe da USF Tatuapé I. Após identificação dos idosos cadastrados, iniciaram-se as visitas domiciliares para a coleta de dados mediante levantamento de nome e endereço dos idosos cadastrados nas listas dos Agentes Comunitários de Saúde, sendo possível realizar entrevista com 55 idosos.

Participaram da pesquisa pessoas que se enquadravam nos critérios de inclusão: idade igual ou superior a 60 anos, fazer parte da região, pertencente ao grupo seis em vulnerabilidade, ser cadastrado na USF de referência e apresentar capacidade de compreensão e comunicação verbal mediante entendimento das questões da entrevista. Enquanto critérios de exclusão: pessoas idosas com doenças ou sequelas que impedissem a realização dos testes (déficits motores graves, auditivos ou afasia); pessoas idosas que fizessem o uso de cadeira de rodas e idosos com doenças em estágio terminal. A coleta de dados se deu por meio de entrevistas domiciliares e foi realizada no período de julho a outubro de 2018, por uma discente devidamente treinada do Curso de Graduação em Gerontologia da Universidade Federal de São Carlos.

A caracterização sociodemográfica da população teve como objetivo apresentar dados referentes a gênero, idade, escolaridade, renda, ocupação, etnia, estado conjugal e se mora com mais alguma pessoa.

Para avaliar a fragilidade utilizou-se: Escala de Fragilidade de Edmonton, Tilburg Frail Scale e Fragilidade Autorreferida. A Escala de Fragilidade de Edmonton é uma escala multidimensional e avalia nove domínios: cognição, estado geral de saúde, independência funcional, suporte social, uso de medicamentos, nutrição, humor, continência e desempenho funcional, investigados por 11 itens. A pontuação máxima é de 17 pontos e representa o nível mais elevado de fragilidade. Os escores para análise da fragilidade são: 0-4, não apresenta fragilidade; 5-6, aparentemente vulnerável; 7-8, fragilidade leve; 9-10, fragilidade moderada; 11 ou mais, fragilidade severa ${ }^{15}$.

A Fragilidade Autorreferida segundo Nunes $(2015)^{16}$ foi investigada segundo cinco critérios, sendo eles: perda de peso, diminuição da atividade física, força de preensão palmar, velocidade da caminhada e fadiga. O resultado da escala é verificado segundo a presença de três ou mais critérios, significando presença de fragilidade, um ou dois critérios indicando pré-fragilidade e nenhum critério, não frágil.

O Tilburg Frailty Indicator é constituído por duas partes, A e B. A parte A é voltada para os determinantes da fragilidade; enquanto, a B referese à identificação da fragilidade propriamente dita, sendo o foco deste estudo. Essa parte é constituída por 15 questões objetivas, autorreferidas, distribuídas em três domínios: físico, psicológico e social. O resultado final é um escore que varia de 0 a 15 pontos. Maior pontuação significa maior nível de fragilidade, ou, alternativamente, escores $>5$ pontos indicam que o indivíduo é frágil ${ }^{17}$. 
Para a avaliação do suporte social utilizouse o Medical Outcomes Study, instrumento que avalia cinco dimensões: apoios material, afetivo, emocional, informação e interação social positiva. Os escores para cada dimensão variam de 20 a 100 pontos. Quanto maior a pontuação, maior o nível de apoio social ${ }^{18}$. Utilizou-se uma questão aberta em relação ao número de pessoas que poderiam auxiliar o idoso em caso de necessidade, sendo esta: "Aproximadamente, quantos amigos intimos ou familiares próximos você tem?.”

Os dados deste estudo foram codificados e organizados em banco de dados em dupla entrada e comparação dos valores, utilizando-se o programa Excel Microsoft. O tratamento e a análise dos dados foram realizados pelo programa estatístico SPSS 20.0 para Windows (Statistical Package for the Social Science). Utilizou-se estatística descritiva, como medidas de tendência central (frequência simples, média, mediana, mínimo e máximo) e medidas de dispersão (desviopadrão). Para avaliar a distribuição normal dos dados utilizaram-se testes não paramétricos como Mann-Whitney e Kruskal-Wallis. A correlação foi verificada segundo Spearman. O nível de significância adotado para os testes estatísticos foi de $5 \%(\mathrm{p} \leq 0,05)$.

O projeto foi submetido e aprovado pelo Comitê de Ética em Pesquisa em Seres Humanos da Universidade Federal de São Carlos e Secretaria Municipal de Saúde de Piracicaba com o Número de Parecer: 2.375.412 e CAAE: 73910317.4.0000.5504, em 10 de novembro de 2017.

\section{Resultados}

Foram entrevistados 55 idosos, sendo a maioria pertencente ao sexo masculino $(57,10 \%)$, com faixa etária entre 60-69 anos $(57,10 \%)$, casados (44,60\%), com baixo nível de escolaridade, 2,85 anos $( \pm 1,12)$ e com uma renda de até um salário mínimo (41,10\%). Aproximadamente $55 \%$ dos entrevistados não eram aposentados e $82,10 \%$ residiam com mais uma pessoa no mesmo domicilio. A idade ( $\mathrm{p}$-valor $<0,0042)$ e a escolaridade ( $p$-valor $<0,001)$ dos participantes se apresentaram enquanto variáveis significantes para a amostra estudada.

A fragilidade foi avaliada com três instrumentos e foi possível observar que nas três avaliações a maioria dos idosos apresentou fragilidade. $\mathrm{Na}$ Fragilidade medida segundo a Escala de Fragilidade de Edmonton, 51,3\% dos respondentes pontuaram para algum nível de fragilidade, na Fragilidade Autorreferida 44,60\% apresentaram fragilidade e na Tilburg Frail Indicator, 75,00\% também apresentaram fragilidade. Apenas para a fragilidade avaliada segundo o Tilburg mostrouse significância em relação à amostra pesquisada (p-valor $<0,009)$.

Com relação ao suporte social, os participantes do estudo tinham ao menos uma pessoa com quem contar em caso de necessidade e em sua maioria demonstram ter apoio social uma vez que, de acordo com a pontuação do instrumento MOS apresentaram uma média 70,47 $( \pm 21,60)$ pontos. No entanto, as dimensões do MOS que apresentaram valores mais baixos foram apoio emocional, com média de $68,27( \pm 27,90)$ pontos e apoio de informação, com média de 69,18 $( \pm 26,33)$ pontos, embora houve significância para todas as dimensões avaliadas, como pode ser verificado na Tabela 1 .

Tabela 1 - Distribuição das variáveis sociodemográficas, de fragilidade e de suporte social de idosos cadastrados em uma Unidade de Saúde da Família. 2018.

\begin{tabular}{llcccccc}
\hline \multicolumn{1}{c}{ Variáveis } & Categorias & n (\%) & Média (dp) & Mínimo & Mediana & Máximo & p-valor** \\
\hline $\begin{array}{l}\text { Dados } \\
\text { numéricos }\end{array}$ & Idade & 55 & $70,15(7,58)$ & 60 & 68 & 91 & $<\mathbf{0 , 0 4 2}$ \\
Gênero & Feminino & $23(41,10)$ & & & & &
\end{tabular}




\begin{tabular}{|c|c|c|c|c|c|c|c|}
\hline Variáveis & Categorias & n (\%) & Média (dp) & Mínimo & Mediana & Máximo & p-valor** \\
\hline & Masculino & $32(57,10)$ & & & & & \\
\hline \multirow[t]{3}{*}{ Idade } & $60-69$ & $32(57,10)$ & & & & & \\
\hline & $70-79$ & $16(28,60)$ & & & & & \\
\hline & $80-100$ & $7(12,50)$ & & & & & \\
\hline \multirow[t]{3}{*}{ Etnia } & Branco & $17(30,40)$ & & & & & \\
\hline & Negro & $13(23,20)$ & & & & & \\
\hline & Outros & $24(42,90)$ & & & & & \\
\hline \multirow[t]{5}{*}{ Estado civil } & Casado & $25(44,60)$ & & & & & \\
\hline & Viúvo & $20(35,70)$ & & & & & \\
\hline & Separado & $5(8,90)$ & & & & & \\
\hline & Divorciado & $2(3,60)$ & & & & & \\
\hline & Não respondeu & $3(5,4)$ & & & & & \\
\hline \multirow[t]{2}{*}{$\begin{array}{l}\text { Mora com } \\
\text { alguém }\end{array}$} & Sim & $46(82,1)$ & & & & & \\
\hline & Não & $7(12,5)$ & & & & & \\
\hline \multirow[t]{2}{*}{$\begin{array}{l}\text { Ocupação } \\
\text { atual }\end{array}$} & Aposentados & $23(41,10)$ & & & & & \\
\hline & Não aposentados & $31(55,40)$ & & & & & \\
\hline \multirow[t]{4}{*}{ Escolaridade } & $\begin{array}{l}\text { Anos de } \\
\text { Escolaridade }\end{array}$ & 52 & $\begin{array}{c}2,85 \\
(1,12)\end{array}$ & 1 & 3 & 6 & $<0,001$ \\
\hline & $\begin{array}{l}\text { Fundamental } \\
\text { Incompleto }\end{array}$ & $39(69,60)$ & & & & & \\
\hline & $\begin{array}{l}\text { Fundamental } \\
\text { Completo }\end{array}$ & $9(16,10)$ & & & & & \\
\hline & $\begin{array}{l}\text { Colegial } \\
\text { Incompleto }\end{array}$ & $4(7,20)$ & & & & & \\
\hline \multirow[t]{5}{*}{ Renda } & $1 \mathrm{SM}^{*}$ & $23(41,10)$ & & & & & \\
\hline & $1-2 \mathrm{SM}$ & $16(28,60)$ & & & & & \\
\hline & 2-3 SM & $8(14,30)$ & & & & & \\
\hline & 3-5 SM & $1(1,80)$ & & & & & \\
\hline & Não sabe & $1(1,80)$ & & & & & \\
\hline \multirow[t]{4}{*}{ EFE } & & $55(100)$ & & & & & $<0,67$ \\
\hline & Não frágil & $12(21.4)$ & & & & & \\
\hline & $\begin{array}{l}\text { Aparentemente } \\
\text { vulnerável }\end{array}$ & $12(21.4)$ & & & & & \\
\hline & Frágil & $31(55.3)$ & & & & & \\
\hline \multirow[t]{2}{*}{ Autorreferida } & & $55(100)$ & & & & & $<0,006$ \\
\hline & Não frágil & $8(14.3)$ & & & & & \\
\hline
\end{tabular}




\begin{tabular}{|c|c|c|c|c|c|c|c|}
\hline Variáveis & Categorias & n (\%) & Média (dp) & Mínimo & Mediana & Máximo & p-valor*** \\
\hline & Pré-frágil & $22(39.3)$ & & & & & \\
\hline & Frágil & $25(44.6)$ & & & & & \\
\hline \multirow[t]{3}{*}{ Tilburg } & & $55(100)$ & & & & & $<0,009$ \\
\hline & Não frágil & $13(23.2)$ & & & & & \\
\hline & Frágil & $42(75.0)$ & & & & & \\
\hline \multirow[t]{6}{*}{ MOS } & & 56 & $\begin{array}{l}70.47 \\
(21.6)\end{array}$ & 0 & 71.5 & 95 & $<0,006$ \\
\hline & Apoio Material & 56 & $86(19.44)$ & 0 & 95 & 100 & $<0,001$ \\
\hline & Apoio Afetivo & 56 & $\begin{array}{c}77.29 \\
(29.31)\end{array}$ & 0 & 96.5 & 100 & $<0,001$ \\
\hline & Apoio Emocional & 56 & $\begin{array}{c}68.27 \\
(27.90)\end{array}$ & 0 & 70 & 100 & $<0,001$ \\
\hline & Apoio Interação & 56 & $\begin{array}{c}70.91 \\
(25.73)\end{array}$ & 0 & 70 & 100 & $<0,001$ \\
\hline & $\begin{array}{l}\text { Apoio } \\
\text { Informação }\end{array}$ & 56 & $\begin{array}{l}69.18 \\
(26.33)\end{array}$ & 0 & 77.5 & 100 & $<0,001$ \\
\hline $\begin{array}{l}\text { Quantidade de } \\
\text { pessoas para } \\
\text { contar }\end{array}$ & & 56 & $\begin{array}{c}1.42 \\
(2.11)\end{array}$ & 0 & 0 & 0 & $<0,001$ \\
\hline
\end{tabular}

Nota: EFE: Edmonton Frail Scale; Tilburg: Tilburg Frail Indicator; MOS: Medical Outcomes Study; SM: Salário Mínimo Legenda: ${ }^{\star S a l a ́ r i o ~ m i ́ n i m o ~ v i g e n t e ~ n o ~ p e r i ́ o d o ~ d a ~ p e s q u i s a: ~} \pm \mathrm{R} \$ 980 ;{ }^{* *} \mathrm{p}$-valor comparação de grupos.

Quando realizada a correlação entre as medidas de fragilidade e o suporte social, não houve indicação de correlação, uma vez que as relações não apresentaram significância, conforme observado na Tabela 2.

Tabela 2 - Correlação entre a fragilidade e o suporte social de idosos cadastrados em uma Unidade de Saúde da Família.

\begin{tabular}{|lcc|}
\multicolumn{1}{c}{ MOS } & \multicolumn{1}{c}{$\mathbf{r}^{*}$} & p-valor \\
\hline EFE & $-0,0,047$ & 0,734 \\
\hline Autorreferida & 0,862 & 0,024 \\
\hline Tilburg & $-0,193$ & 0,158 \\
\hline
\end{tabular}

Nota: EFE: Edmonton Frail Scale; MOS: Medical Outcomes Study Legenda: ${ }^{\star}$ Correlação de Spearman

\section{Discussão}

Os participantes do presente estudo foram em sua maioria homens, achado esse que difere de outros estudos com idosos cadastrados em Unidades de Saúde da Família (USF) ${ }^{5,19}$. Em relação à idade, a maioria dos participantes tinham entre 60 e 69 anos, "idosos jovens" e que apresentaram fragilidade. Tal achado demostrada a necessidade de prevenção em relação às condições de saúde e consequentemente à fragilidade na Atenção Básica de Saúde. Evidência em literatura científica tem demostrado idosos jovens apontando para a fragilidade em contexto de vulnerabilidade social $^{20}$. A maioria dos entrevistados era casado e com ensino fundamental incompleto o que 
corrobora com o estudo de Neves $(2018)^{5}$ e de Fernandes (2013) ${ }^{19}$, ambos realizados em amostras e contextos semelhantes a desse estudo.

No presente estudo, $56 \%$ dos idosos avaliados com a Escala de Fragilidade de Edmonton (EFE) pontuaram para algum nível de fragilidade. Em estudo de Fernandes et al $(2013)^{19}, 30,1 \%$ dos idosos avaliados $(\mathrm{n}=128)$ estavam frágeis avaliados em serviço de atenção básica e contexto de vulnerabilidade social. Em estudo de Carneiro et al $(2017)^{21}$, ao avaliar 360 idosos atendidos em um Centro de Referência do Idoso com a EFE, a prevalência de fragilidade foi $47,2 \%$.

No presente estudo a fragilidade Autorreferida indicou que $44,6 \%$ dos participantes estavam frágeis. Esse valor ficou próximo aos achados do estudo de Llano et al (2017) ${ }^{22}$ que avaliaram 820 idosos residentes em áreas rurais e constatouse que $43,4 \%$ dos entrevistados apresentaram fragilidade.

Para a fragilidade avaliada com o Tilburg Frailty Indicator o presente estudo indicou que $75 \%$ dos participantes estavam frágeis. O estudo realizado por Neves et al $(2018)^{5}$, com 377 idosos usuários de Estratégia de Saúde da Família, identificaram uma prevalência de fragilidade de $65,25 \%$.

A fragilidade avaliada, segundo instrumentos subjetivos neste estudo, corroborou com achados em literatura científica. $\mathrm{O}$ uso de instrumentos subjetivos para avaliação da fragilidade tem vantagens por serem multidimensionais, de rápida avaliação, com baixo custo, foram validados com idosos da comunidade e podem ser aplicados por diferentes profissionais. Ademais, o uso de instrumentos subjetivos para rastreamento da fragilidade pode ser adotado por equipes de atenção básica à saúde ${ }^{16,23}$.

Para este estudo optou-se verificar a fragilidade somente por instrumentos subjetivos e multidimensionais. Os idosos avaliados neste estudo apresentaram fragilidade para os três instrumentos. Estudos em regiões de vulnerabilidade social têm apresentado a presença de fragilidade com instrumentos multidimensionais; há evidencia que o contexto de moradia pode ser um determinante e esteja associado à presença de fragilidade ${ }^{4}$. No entanto, diferentes contextos regionais e sociais poderiam influenciar na prevalência da fragilidade e se observar fragilidade em idosos em contextos de vulnerabilidade social, ${ }^{24}$ como se observou no presente estudo.

Com relação ao apoio social, os resultados apontam que os idosos têm apoio social e podem contar com outras pessoas. Acerca do suporte social, a literatura científica destaca que pessoas frágeis, com poucos contatos sociais têm aumentado os desfechos adversos como mortalidade, necessidade de cuidados prolongados e hospitalizações recorrentes ${ }^{25}$.

Guedes e Colaboradores $(2017)^{26}$, em ensaio a respeito do apoio social em relação à saúde da pessoa idosa, afirmam que a rede de suporte social deve não apenas permitir o apoio familiar, de amigos e da vizinhança, senão que também deve permitir a interação do idoso nessa rede. Os Autores ainda discutem diversas estratégias para abordagem da rede como grupos de idosos, participação em voluntariado, uso de redes sociais informatizadas, trabalhos intergeracionais em escolas, entre outros.

O presente estudo não encontrou correlação entre fragilidade e suporte social, a baixa oferta de apoio e relações sociais podem ser um fator de risco para a fragilidade e consequentemente levar a óbito ${ }^{27}$. Dado esse relevante, uma vez que idosos podem se tornar menos ativos socialmente se não contarem com uma rede de apoio familiar e social e este fator ser preditor para a fragilidade ${ }^{27}$. No entanto, ter suporte social promove efeito protetor como o bem-estar físico e psicológico e pode ser associado às melhores condições de saúde ${ }^{27}$. Embora as correlações não foram evidenciadas neste estudo, outras pesquisas confirmam esse achado com amostras maiores. ${ }^{28,29}$

Este estudo apresenta vantagens e limitações. A principal vantagem é que pode nortear estratégias para futuras ações preventivas na atenção básica de saúde para a população residente em áreas vulneráveis. Um planejamento da assistência pelos profissionais de saúde, direcionamento para os serviços de saúde da rede e organização dos serviços pelos gestores podem ser efetivos quando há o rastreamento de saúde da pessoa idosa, por meio de instrumentos subjetivos e, assim, prever formas de ações direcionadas para idosos por meio do plano terapêutico singular e planejamento da linha de cuidado a longo prazo. Esses resultados 
podem indicar a necessidade de atenção nas redes de apoio da família ou dos serviços sociais e de saúde para esses idosos participantes do estudo.

As limitações deste estudo estão relacionadas ao tamanho amostral e à realização do estudo em uma única região de vulnerabilidade social, estudos que incluam outros contextos poderão enriquecer achados quanto à relação da fragilidade com o suporte social.

\section{Conclusões}

O estudo não apresentou relação entre fragilidade e suporte social. O presente estudo avaliou a fragilidade do idoso com instrumentos subjetivos de rápida aplicação que podem ser adotados pelas equipes de atenção primária à saúde, em região de vulnerabilidade social. Destaca-se que a fragilidade é uma condição de saúde prevalente em pessoas idosas e é acometida por diferentes fatores, sendo um deles a ausência ou a presença de suporte social. No entanto rastrear tais condições pode direcionar estratégias preventivas pelas equipes de atenção primária à saúde visando cuidado de longo prazo.

\section{Referências}

1. de Melo LA, Ferreira LM, dos Santos MM, de Lima KC. Fatores socioeconômicos, demográficos e regionais associados ao envelhecimento populacional. Revista Brasileira de Geriatria e Gerontologia. 2017;20(4):494502.

2. Morley JE, Vellas B, Van Kan GA, Anker SD, Bauer JM, Bernabei R, Cesari M, Chumlea WC, Doehner W, Evans J, Fried LP. Frailty consensus: a call to action. Journal of the American Medical Directors Association. 2013 Jun $1 ; 14(6): 392-7$

3. Lourenço RA, Moreira VG, Mello RG, Santos ID, Lin SM, Pinto AL, Lustosa LP, Duarte YA, Ribeiro JA, Correia CC, Mansur HN. Consenso brasileiro de fragilidade em idosos: conceitos, epidemiologia e instrumentos de avaliação. Geriatrics, Gerontology and Aging. 2018;12(2):121-35.

4. de Jesus IT, dos Santos Orlandi AA, Zazzetta MS. Fragilidade e apoio social e familiar de idosos em contextos de vulnerabilidade. Revista da Rede de Enfermagem do Nordeste. 2018;19.
5. Neves ÁD, Silva AM, Cabral JF, Mattos IE, Santiago LM. Prevalence of and factors associated with frailty in elderly users of the Family Health Strategy. Revista Brasileira de Geriatria e Gerontologia. 2018 Dec;21(6):680-90.

6. Llano PM, Lange C, Nunes DP, Pastore CA, Pinto AH, Casagranda LP. Fragilidade em idosos da zona rural: proposta de algoritmo de cuidados. Acta Paul. Enferm. (Online). 2017;30(5):520-30.

7. Cesari M, Prince M, Thiyagarajan JA, De Carvalho IA, Bernabei R, Chan P, Gutierrez-Robledo LM, Michel JP, Morley JE, Ong P, Manas LR. Frailty: an emerging public health priority. Journal of the American Medical Directors Association. 2016 Mar 1;17(3):188-92.

8. Rodrigues NO, Neri AL. Vulnerabilidade social, individual e programática em idosos da comunidade: dados do estudo FIBRA, Campinas, SP, Brasil. Ciência \& Saúde Coletiva. 2012;17:2129-39.

9. Amaral FL, Guerra RO, Nascimento AF, Maciel ÁC. Apoio social e síndrome da fragilidade em idosos residentes na comunidade. Ciência \& Saúde Coletiva. 2013;18:1835-46.

10. Alvarenga MR, Oliveira MA, Domingues MA, Amendola F, Faccenda O. Rede de suporte social do idoso atendido por equipes de Saúde da Família. Ciência \& Saúde Coletiva. 2011;16:2603-11.

11. Marques AK, Landim FL, Collares PM, Mesquita RB. Apoio social na experiência do familiar cuidador. Ciência \& Saúde Coletiva. 2011;16:945-55.

12. Brasil. Ministério da Saúde. Diretrizes para o cuidado das pessoas idosas no SUS. Proposta de modelo de atenção integral. XXX Congresso Nacional de Secretarias Municipais e Saúde. Brasília: Ministério da Saúde, 2014.

13. Brasil. Ministério da Saúde, Secretaria de Atenção à Saúde. Política Nacional de Saúde da Pessoa Idosa. Portaria n²528 de 19 de outubro de 2006. Disponível em: http://bvsms.saude.gov.br/bvs/saudelegis/gm/2006/ prt2528_19_10_2006.html

14. Fundação Seade. Distribuição da população, segundo grupos do Índice Paulista de Vulnerabilidade Social. São Paulo, 2010. Disponível em: http://catalogo. governoaberto.sp.gov.br/dataset/21-ipvs-indice-paulistade-vulnerabilidade-social

15. Fabrício-Wehbe SC, Schiaveto FV, Vendrusculo TR, Haas VJ, Dantas RA, Rodrigues RA. Adaptação cultural e validade da Edmonton Frail Scale-EFS em uma amostra de idosos brasileiros. Revista Latino-Americana de Enfermagem. 2009;17(6).

16. Nunes DP, de Oliveira Duarte YA, Santos JL, Lebrão ML. Rastreamento de fragilidade em idosos por instrumento autorreferido. Revista de Saúde Pública. 2015;49:1-9.

17. Santiago LM, Luz LL, Mattos IE, Gobbens RJ. Adaptação transcultural do instrumento Tilburg Frailty Indicator (TFI) para a população brasileira. Cadernos de Saúde Pública. 2012;28:1795-801. 
18. Andrade CR. Associaçäo entre apoio social e freqüência relatada de auto-exame das mamas no estudo prósaúde (Doctoral dissertation).

19. Fernandes HD, Gaspar JC, Yamashita CH, Amendola F, Alvarenga MR, Oliveira MA. Avaliação da fragilidade de idosos atendidos em uma unidade da estratégia saúde da família. Texto \& Contexto-Enfermagem. 2013 Jun;22(2):423-31.

20. Zazzetta MS, Gomes GA, Orlandi FS, Gratão AC, Vasilceac FA, Gramani-Say K, Ponti MA, Castro PC, Pavarini SC, Menezes AL, Nascimento CM. Identifying frailty levels and associated factors in a population living in the context of poverty and social vulnerability. J Frailty Aging. 2017 Jan 1;6(1):29-32.

21. Carneiro JA, Cardoso RR, Durães MS, Guedes MC, Santos FL, Costa FM, Caldeira AP. Frailty in the elderly: prevalence and associated factors. Revista brasileira de enfermagem. 2017 Aug;70(4):747-52.

22. Llano PM, Lange C, Nunes DP, Pastore CA, Pinto AH, Casagranda LP. Fragilidade em idosos da zona rural: proposta de algoritmo de cuidados. Acta Paul. Enferm. (Online). 2017;30(5):520-30.

23. Rajabali, Naheed, Darryl Rolfson, and Sean M. Bagshaw. Assessment and utility of frailty measures in critical illness, cardiology, and cardiac surgery. Canadian Journal of Cardiology. 2016;32(9):1157-1165.

24. Andrew MK. Frailty and social vulnerability. InFrailty in Aging 2015 (Vol. 41, pp. 186-195). Karger Publishers.

25. Mulasso A, Roppolo M, Giannotta F, Rabaglietti E. Associations of frailty and psychosocial factors with autonomy in daily activities: a cross-sectional study in Italian community-dwelling older adults. Clinical interventions in aging. 2016;11:37.

26. Guedes MB, Lima KC, Caldas CP, Veras RP. Apoio social e o cuidado integral à saúde do idoso. Physis: Revista de Saúde Coletiva. 2017;27:1185-204.

27. de Brito TR, Nunes DP, Corona LP, da Silva Alexandre T, de Oliveira Duarte YA. Low supply of social support as risk factor for mortality in the older adults. Archives of gerontology and geriatrics. 2017 Nov 1;73:77-81.
28. Andrade JM, Duarte YA, Alves LC, Andrade FC, Souza PR, Lima-Costa MF, Andrade FB. Perfil da fragilidade em adultos mais velhos brasileiros: ELSI-Brasil. Rev. Saúde Pública. 2018 Sep 28;52(Suppl 2).

29. Ye B, Gao J, Fu H. Associations between lifestyle, physical and social environments and frailty among Chinese older people: a multilevel analysis. BMC geriatrics. 2018 Dec;18(1):314.

\section{Como citar este artigo:}

Moura K, Jesus ITM, Orlandi AAS, Zazzetta MS. Fragilidade e suporte social de idosos em região vulnerável: uma abordagem em uma unidade de saúde da família. Rev. Aten. Saúde. 2020; 18(63): 65-73. 